\title{
Review Article \\ DNA Nanobiosensors: An Outlook on Signal Readout Strategies
}

\author{
Arun Richard Chandrasekaran \\ Confer Health, Inc., Charlestown, MA, USA \\ Correspondence should be addressed to Arun Richard Chandrasekaran; arunrichard@nyu.edu
}

Received 18 January 2017; Revised 1 May 2017; Accepted 9 May 2017; Published 30 May 2017

Academic Editor: Jorge Pérez-Juste

Copyright (C) 2017 Arun Richard Chandrasekaran. This is an open access article distributed under the Creative Commons Attribution License, which permits unrestricted use, distribution, and reproduction in any medium, provided the original work is properly cited.

\begin{abstract}
A suite of functionalities and structural versatility makes DNA an apt material for biosensing applications. DNA-based biosensors are cost-effective and sensitive and have the potential to be used as point-of-care diagnostic tools. Along with robustness and biocompatibility, these sensors also provide multiple readout strategies. Depending on the functionality of DNA-based biosensors, a variety of output strategies have been reported: fluorescence- and FRET-based readout, nanoparticle-based colorimetry, spectroscopy-based techniques, electrochemical signaling, gel electrophoresis, and atomic force microscopy.
\end{abstract}

\section{Introduction}

Biosensing is an area of research that has garnered attention due to its importance in medical diagnostics, biomolecular analysis, and studies involving molecular pathways. A variety of nanomaterials such as quantum dots, gold and silver nanoparticles, metallic nanowires, and carbon nanotubes have been developed for sensing applications [1-3]. These materials provide unique optical, electronic, chemical, and mechanical properties and contribute to both robust sensing and convenient and sensitive readout strategies. DNA is one other material that has found applications in biosensing. Over the past three decades, DNA has become a versatile material for bottom-up nanofabrication of two- [4] and threedimensional lattices [5], nanoscale objects [6], and complex wireframe structures [7] with applications in biology [8], medicine [9], and materials science [10]. The structural aspects of DNA nanostructures combined with their ability to respond to stimuli have led to the creation of cost-effective and sensitive biosensors that are functional under a wide range of biologically relevant temperatures and conditions [11-14]. The recognition event in such biosensing approaches can be translated by specific readout strategies which are briefly discussed in this article in the following categories: (i) fluorescence, (ii) FRET, (iii) nanoparticle-based color change, (iv) electrochemical signaling, (v) gel electrophoresis, (vi) atomic force microscopy (AFM), and (vii) surface-enhanced Raman spectroscopy (SERS).

\section{DNA Nanostructures for Biosensing}

The field of DNA nanotechnology has seen the development of dynamic DNA machines [15] and devices [16] that respond to chemical or environmental stimuli. This concept is extended to DNA-based biosensors that rely on specific recognition events between a substrate and the target analyte (e.g., nucleic acid and protein detection) or programmed conformational changes (e.g., $\mathrm{pH}$ sensing). DNA nanostructures have some advantages for being used in biosensing. The intermolecular recognition of rationally designed DNA sequences allows highly precise design and construction of DNA nanostructures. The nanoscale dimensions of these structures provide large surface-to-volume ratios, thus resulting in large signal changes on target binding. DNA sequences can now be synthesized in large quantities in a cost-effective manner and can be chemically modified to enhance their functionality.

A majority of nucleic acid-based biosensors involve hybridization of a DNA or RNA strand to its complement (Figure 1(a)), or a complementary region in a stem loop (Figure 1(b)). Such stem-loop configurations can also be used to monitor global environmental changes such as changes in temperature by using a fluorophore-quencher pair on the ends of the strands. Biosensors for other stimuli such as $\mathrm{pH}$ changes are based on structures that involve triplex [17] (Figure 1(c)), i-motif formation [18] (Figure 1(d)), or poly-dA helix formation (Figure 1(e)) [19], all of which are 


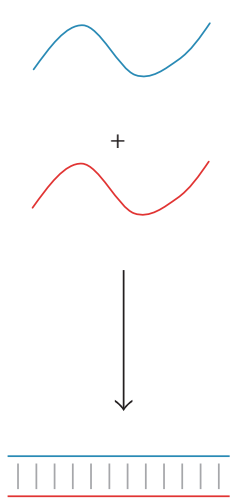

Duplex formation

(a)

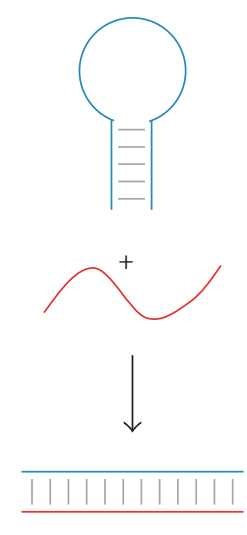

Stem-loop configuration
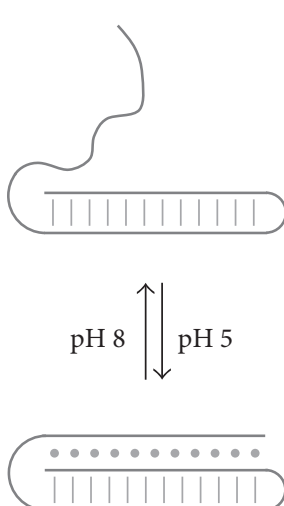

Triplex formation
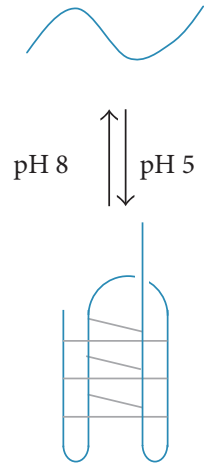

i-motif formation

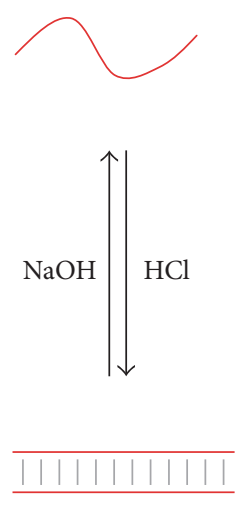

poly dA helix

(e)
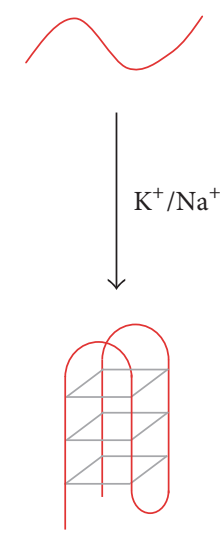

G-quadruplex formation

(f)

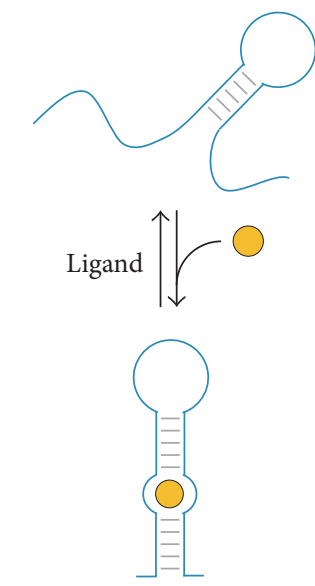

Aptamer-based recognition

(g)

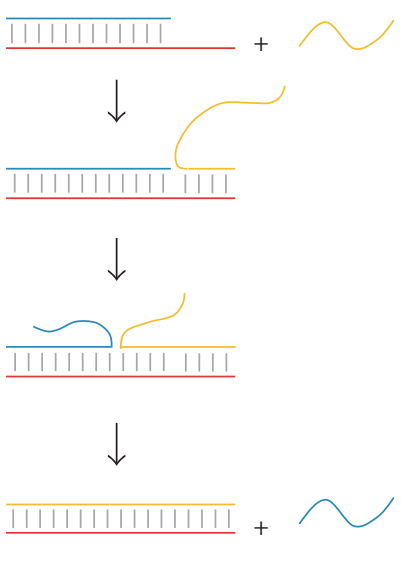

Toehold-based strand displacement

(h)

Figure 1: Concepts used in biosensing platforms. (a, b) Sequence-specific DNA hybridization in a duplex and stem-loop configuration, (c) triplex formation under acidic conditions, (d) i-motif formation under acidic conditions by C-rich DNA strands, (e) poly-dA helix formation under acidic conditions by poly-A strands, (f) G-quadruplex formation in the presence of $\mathrm{K}^{+}$or $\mathrm{Na}^{+}$, (g) aptamer reconfiguration in the presence of specific ligands, and (h) toehold-based strand displacement.

sequence-specific and occur under acidic conditions. Gquadruplex formation is another sequence-specific conformational change that forms under specific ionic conditions such as the presence of $\mathrm{K}^{+}$or $\mathrm{Na}^{+}$[20] (Figure 1(f)). In addition, aptamer-based recognition has also been widely used in biosensors (Figure $1(\mathrm{~g})$ ). These are single-stranded oligonucleotides that can bind with high affinity to ions (e.g., $\mathrm{K}^{+}$, $\mathrm{Hg}^{2+}$, and $\mathrm{Pb}^{2+}$ ), small organic molecules (e.g., ATP, amino acids, and vitamins), peptides, proteins (e.g., thrombin and growth factors), and even whole cells or microorganisms (e.g., bacteria) [21, 22], resulting in a secondary structure formation. Toehold-based strand displacement [23] (Figure 1(h)) has also been used for target readouts and signal amplification in many DNA-based biosensing platforms.
2.1. Fluorescence-Based. Conformational changes in a DNA nanostructure can be observed by tagging the component DNA strands with a fluorophore-quencher pair. For example, if a fluorophore and quencher are attached to two ends of a single strand forming a stem loop, the fluorescence is quenched in the stem-loop configuration due to the close proximity $(<10-100 \AA)$ of the fluorophore to the quencher (Figure 2(a), top). Change in the stem-loop configuration moves the fluorophore away from the quencher, thus increasing the fluorescence. This strategy has been used in a DNAbased beacon for the detection of antibodies and proteins [24]. This molecular switch was composed of a stem-loop system comprising a long strand that contained the loop and two short complementary strands with single-stranded tails. The ends of these tails were modified to contain an 


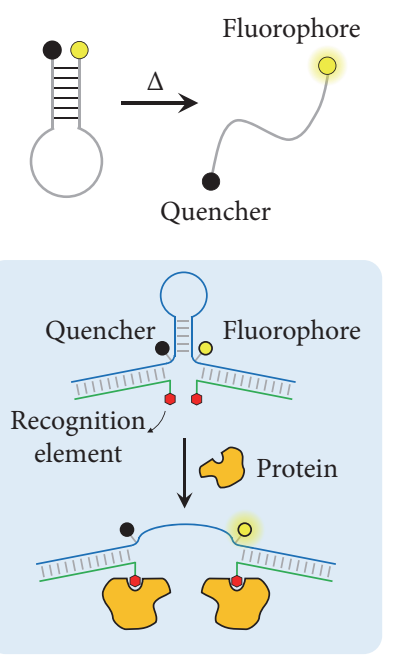

(a)

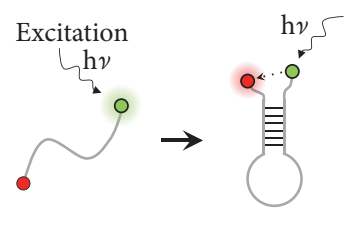

Open state: Low FRET

ar

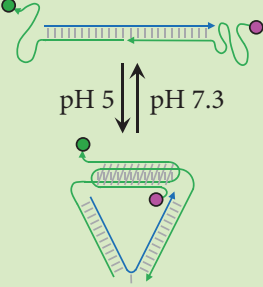

Closed: High FRET
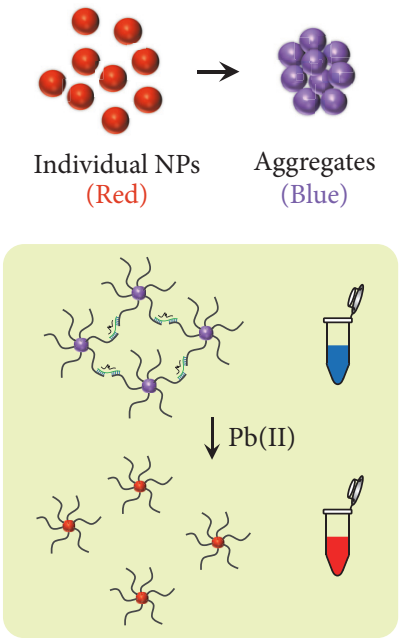

(c)
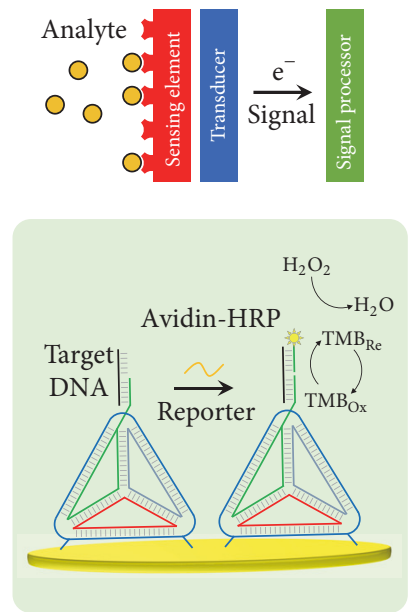

(d)
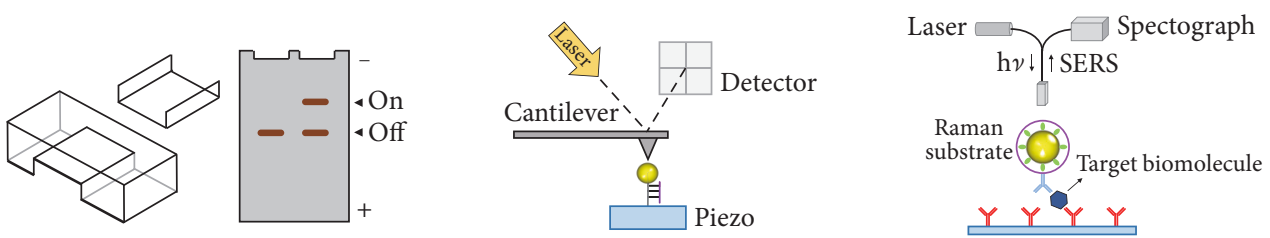

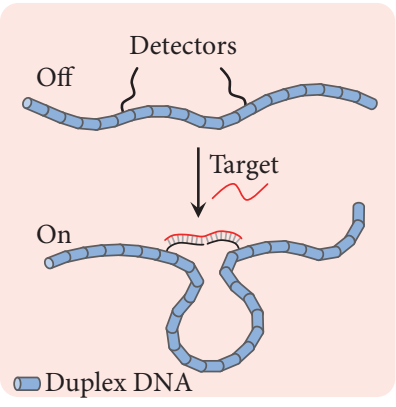

(e)

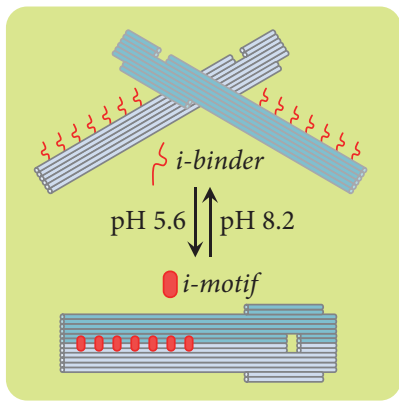

(f)

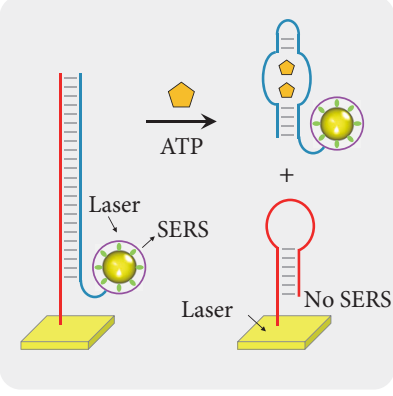

(g)

FIGURE 2: Readout strategies for DNA nanostructure-based biosensors. (a) Fluorescence-based readout: the example shown demonstrates the detection of proteins resulting in stem-loop reconfiguration leading to a fluorescent signal [24]. (b) FRET-based readout: The example shows a DNA nanodevice containing a FRET pair on opposite ends of a nicked duplex. The C-rich single-stranded extensions on either end of the duplex can form an i-motif at low pH resulting in a FRET signal [18]. (c) NP-based color change: nanoparticles aggregated via DNA strands and a DNAzyme are blue; presence or addition of $\mathrm{Pb}^{2+}$ ions causes cleavage of the DNAzyme resulting in nanoparticle disaggregation and a change in color to red [25]. (d) Electrochemical readout: DNA tetrahedra with single-stranded pendants can bind partially to target DNA. The remainder of the target DNA strand can bind a reporter strand that produces a HRP-based electrochemical readout [26]. (e) Gel electrophoresis: a DNA nanoswitch containing two single-stranded overhangs that are partially complementary to target DNA. Binding of target DNA to the two detectors causes the linear "off" state to change into a looped "on" state. The two states of the nanoswitch migrate differently on a gel, thus providing a digital on-off signal [27]. (f) AFM-based readout: DNA origami levers that contain C-rich single-stranded extensions can act as $\mathrm{pH}$ sensors. In acidic $\mathrm{pH}$, the single-stranded extensions on each half of the lever can form an intermolecular i-motif causing a conformational change that can be visualized on an AFM [28]. (g) SERS-based readout: an ATP-binding aptamer is bound to a single-stranded probe on a gold surface. The presence of ATP triggers conformational change of the aptamer causing it to dissociate from the probe, resulting in a loss of the SERS signal [29].

appropriate target-specific recognition element (e.g., digoxigenin). The stem region contained a fluorophore/quencher pair and the fluorescent signal is quenched as long as the stem remains closed (Figure $2(\mathrm{a})$, bottom). Binding of a dig-specific antibody to both recognition elements (bivalent binding) pushes them away, thus opening the stem region and in turn causing enhanced fluorescence. This sensor was used to detect a variety of antibodies and protein targets including the HIV biomarker anti-p17 antibody.

Fluorescence-based biosensors have also been used for $\mathrm{pH}$ detection. One such example involves C-rich DNA strands containing a fluorophore on one end attached to a gold surface [30]. At acidic $\mathrm{pH}$, the strands form an intramolecular i-motif, bringing the fluorophore closer to the 
gold surface and essentially quenching it. At basic $\mathrm{pH}$, the single strands can bind to a complementary strand pushing the fluorophore away from the gold surface, thereby enhancing the fluorescence. A similar example used a graphene surface instead of gold and worked on the basis of $\mathrm{pH}$ dependent triplex formation [31]. In addition, solution-based triplex-forming nanoswitches have also been developed for $\mathrm{pH}$ detection [17]. This switch was designed so that the fluorophore-quencher pair remains closer when the switch forms a triplex, acting as an indicator of the $\mathrm{pH}$ range. Another example of fluorescence-based readout was DNAtweezer nanostructures that were designed to contain restriction sites specific to endonucleases [32]. The presence of these endonucleases causes cleavage of component strands resulting in an increased fluorescent signal.

2.2. FRET-Based. Structural transitions in DNA nanostructures have been analyzed using Fluorescence Resonance Energy Transfer (FRET), in which fluorescence signals are generated for molecular association and separation in the $1-10 \mathrm{~nm}$ range (Figure 2(b), top) [33]. One such example is a DNA nanomachine based on an intramolecular i-motif that has been used as a $\mathrm{pH}$ sensor inside living cells [18]. The basis of this machine was a conformational change from an open linear structure under physiological conditions $(\mathrm{pH}$ 7.3, low FRET) to a closed triangular structure under acidic conditions ( $\mathrm{pH}$ 5.0, high FRET) (Figure 2(b), bottom). This switch was effective in $\mathrm{pH}$ ranges 5.5 to 6.8 and was used to map spatial and temporal $\mathrm{pH}$ changes associated with endosome maturation in Drosophila hemocytes [18] as well as inside a multicellular organism (Caenorhabditis elegans) [34]. A similar strategy was also used to simultaneously map the $\mathrm{pH}$ gradients along two different but intersecting endocytic pathways inside the same cell [35]. In another example, the edges of a DNA tetrahedron were designed to contain dynamic sequences that are specific to adenosine triphosphate (ATP) [36]. These regions undergo a conformational change in the presence of ATP and were used to detect intracellular ATP via a FRET signal.

2.3. Nanoparticle-Based. Metallic nanoparticles (NPs) have been shown to exhibit defined color changes between individual nanoparticles and aggregated clusters [37, 38]. This characteristic has been used in colorimetric assays based on DNAfunctionalized gold nanoparticles (AuNPs) that provide an optical readout (visual color change) (Figure 2(c), top). One such example is the DNAzyme-mediated self-assembly of AuNPs that was used in the detection of $\mathrm{Pb}$ (II) ions [25]. The DNAzyme used in this case consists of an enzyme strand and a substrate strand. The substrate strand was extended on both ends with sequences complementary to the single strands on the AuNPs. Hybridization of these two DNA strands results in aggregation of the AuNPs (blue color). On addition of $\mathrm{Pb}$ (II), the enzyme strand catalyzes cleavage of the substrate strand resulting in disaggregation of the AuNPs, thereby resulting in a color change to red (Figure 2(c), bottom). This visual color change acts as an indicator of the presence or absence of $\mathrm{Pb}(\mathrm{II})$ ions. Another example that uses NP-based visual color change is a lateral flow nucleic acid biosensor for detecting nucleic acid sequences [39]. In this case, AuNPs were modified to contain biotin-tagged DNA strands that were complementary to a target DNA strand. Target-bound DNA-AuNP conjugates get accumulated on a streptavidin coated test line, thus resulting in a color change (red) of the test line.

2.4. Electrochemical Readout. DNA-based electrochemical sensors use nanoscale interactions between the target and a recognition layer and the signal is transduced (e.g., by enzyme activity) via a solid electrode surface (Figure 2(d), top). DNA tetrahedron-based biosensors combined with surface-based assays have been used for electrochemical detection of nucleic acids [26]. In this design, the bottom three vertices of the tetrahedral DNA probe were bound to a gold electrode surface via thiol modifications. The fourth vertex was designed to contain a DNA strand that is complementary to part of the target (Figure 2(d), bottom). When part of the target binds to the probe, a biotinylated reporter probe binds to the remaining part of the target. This hybridization event is then transduced into electrochemical signals through the specific binding of an avidin-HRP (horseradish peroxidase) conjugate to the biotin, leading to enzyme turnover-based signal transduction. A similar strategy, combined with multibranched hybridization chain reaction (mHCR) for improved sensitivity, was used for cancer cell detection [40]. Moreover, by conjugating the DNA tetrahedral probe to an antibody (e.g., tumor necrosis factor alpha), the strategy has been redesigned for immunological sensing as well [41]. In another example, electrochemical sensing of HIV DNA has been done using long-range self-assembled DNA constructs [42]. In this case, the output signal was based on the accumulation of hexaammineruthenium(III) chloride (RuHex) on the negatively charged phosphate backbone of the DNA via electrostatic forces.

Electrochemical aptamer-based sensors have been developed for the detection of proteins, small molecules, and inorganic ions [43]. In this case, an aptamer probe containing an electrochemical redox reporter molecule is attached onto a gold electrode. Target binding induces a conformational change of the aptamer, thus altering the position of the reporter relative to the electrode, yielding a measurable current change. Such a strategy has been used to construct a realtime biosensor capable of continuously tracking doxorubicin (a chemotherapeutic) and kanamycin (an antibiotic) in live rats and in human whole blood $[44,45]$. This strategy has also been used to detect specific proteins [46] and antibodies [47] directly in undiluted blood serum.

2.5. Gel Electrophoresis-Based. Gel electrophoresis is the most ubiquitous technique in a biology or biochemical laboratory (Figure 2(e), top). DNA nanoswitches have been designed for analysis of biomolecular interactions such as biotin-streptavidin, antibody-antigen, peptide ligation, and restriction enzyme cleavage [48]. These events result in a conformational change of the nanoswitch that can be analyzed through gel electrophoresis. This strategy was recently adapted for the detection of specific nucleic acid sequences [27]. The off state of the nanoswitch is a linear duplex formed 
by a single-stranded M13 scaffold and a set of staple strands. Two of the staple strands were modified to contain singlestranded extensions (detectors), each of which binds to parts of the target. Hybridization of the target oligonucleotide to the detectors reconfigures the switch to form a loop, thus changing it to the on state (Figure 2(e), bottom). The "off" and "on" states of the DNA nanoswitches migrate differently on an agarose gel. Gel-shift assays are routinely used in laboratories, and this strategy provides a relatively easy and one-step method to detect target nucleic acids by the appearance of the "on" band.

2.6. AFM-Based. Atomic force microscopy (AFM) is frequently used to analyze two-dimensional constructs made from DNA (Figure 2(f), top). The DNA origami technique [49] provides a convenient route to the assembly of such two-dimensional platforms that allow the arrangement of functional moieties. For example, DNA origami sheets containing single-stranded DNA probes complementary to a target sequence can act as molecular chips for detecting the presence of the target oligonucleotide [50]. Hybridization of the probe tiles to the target in solution was detected using AFM, based on the difference in elastic properties of single-stranded (probes without target) and doublestranded DNA (probes bound to target). AFM readout is more pronounced in structures that change conformation or lead to a visual marker on target interaction. In one such example, DNA origami was used to construct a "nanoplier" containing C-rich sequences (i-binders) on each lever of the plier [28]. Under acidic conditions, these sequences form an intermolecular i-motif, thereby bringing the two levers together (Figure 2(f), bottom). This structural transition can be visualized using AFM. In another example, DNA origami tiles with specific topological markers were used to detect single nucleotide polymorphisms (SNPs) producing a direct visual readout of the target nucleotide contained in the probe sequence [51]. The platform contained graphical representations of the four nucleotides $\mathrm{A}, \mathrm{T}, \mathrm{G}$, and $\mathrm{C}$, and the symbol containing the test nucleotide identity disappears in the presence of the target.

2.7. SERS-Based. Surface-enhanced Raman scattering (SERS), a variation of standard Raman spectroscopy, provides a significantly enhanced Raman signal through electromagnetic interaction between the analyte molecules and metal surface [52, 53]. In typical SERS assays, Raman reporters are attached to the surface of metallic nanoparticles (the SERS substrates) and covered by a protective shell (Figure 2(g), top) that prevents leaching out of the Raman reporters and improves water solubility and stability $[54,55]$. In one such example, an aptamer-based biosensor was designed to detect ATP using SERS [29]. This study used malachite green isothiocyanate as the Raman reporter which was sandwiched between a gold nanostar core and a silica shell. The aptamer probe, specific to ATP, was immobilized on a gold surface by hybridization to complementary single-stranded DNA that is attached to the gold surface (Figure 2(g), bottom). Binding of ATP to the aptamer causes it to fold, thereby detaching from its complementary strand. This duplex dissociation causes a reduction in the SERS signal, thus acting as a detection mechanism for ATP molecules.

Spatial control of plasmonic nanoparticles using rigid DNA nanostructures allows the creation of distinct structuredependent optical features [56]. One such example is the use of a DNA tetrahedron to control the positioning of AuNPs [57]. By using thiol-modified DNA strands that can selfassemble into a tetrahedron, the structure can be used to recruit $20 \mathrm{~nm}$ AuNPs on each of its four vertices. In addition, the DNA tetrahedron was designed to contain Cy3 molecule, a Raman active dye, on one of its edges. This structure was further coated with silver to form Ag-Au nanoshells. The system was used to detect single-stranded DNA that was complementary to the component strands of the tetrahedron. Target addition causes formation of duplexes by hybridization of the component strands with the target, resulting in disassembly of the NP cluster, therefore causing a different SERS signal for the duplexes. Another example is a DNA origami platform that was used to assemble $40 \mathrm{~nm}$ AuNP dimers with sub- $5 \mathrm{~nm}$ gaps between them [58]. The origami platform provides a strong plasmonic coupling between the NPs and this system was used to attain SERS measurements of specific single-stranded DNA molecules. Depending on the sequences of the single-stranded DNA that is coated on the surface of the NP dimers, specific SERS spectral peaks are attained that can be used to detect or identify specific DNA sequences.

\section{Discussion and Outlook}

Multidimensional DNA nanostructures have been shown to be useful as frameworks for precisely programmed arrangement of functional molecules such as ligands, enzymes, and chemical groups $[59,60]$. These strategies involve sequencespecific recognition of a DNA nanostructure [61] or covalent linkage of a functional moiety on the DNA strand [62]. Such site-specific positioning of biomolecules allows these structures to be used as biosensing platforms for a variety of target analytes. Moreover, the ability to design triggered responses to a variety of external chemical and biological stimuli makes DNA-based devices versatile for biosensing. Such stimuli responsive structures can be not only used as biosensors but also configured to react to specific biomarkers and release cargos from macromolecular containers [63]. For example, a recent enzyme-powered DNA-AuNP nanomachine was used to release payloads while also serving as a biosensor for nucleic acid detection [64]. Such nanostructures that can act according to stimuli can be used as "sense-and-treat" devices for theranostic applications [65]. Sensing capabilities can also be combined with computing platforms for this purpose [66].

The dynamic nature of such programmable DNA devices plays a major role in the development of robust and sensitive molecular sensing that is functional at the nanoscale while providing a convenient signal readout. The potential use of a specific biosensing strategy is reliant on factors such as assay/readout time, skill required to perform the assay, the amount of sample required and the dynamic range and sensitivity, and the cost of the method. Considering these factors, the use of AFM potentially limits the practicality 
of such biosensors due to the equipment cost and the requirement of skilled personnel. Furthermore, AFM can only be used to read out surface-based assays or those involving 2D DNA nanostructures and requires visualizing multiple fields of the sample (usually deposited on a mica surface) to yield quantitative results. The gel-based readout such as the one using DNA nanoswitches provides a simple assay for research laboratories to identify the presence of a target nucleic acid without requiring multiple mixing steps or enzymatic amplification. This method is currently limited to laboratory usage but can be extended to point-of-care testing by using bufferless gel systems and portable electrophoresis units. Moreover, the cost of gel-based assays is much cheaper as it only requires already existing equipment in a lab. Nanoparticle-based assays, both optical and SERS, have been very successful in developing point-of-care diagnostics with a relatively easier assay and quicker readout. One notable platform is the lateral flow assay which has been frequently used in clinical diagnostics with a simple visual readout (a colored test line) compared to a standard (a control line). These tests do not require any equipment and provide the end user with a "yes or no" answer.

One limitation of current biosensing strategies is the use of multiple steps for signal generation and amplification which increases the time required for detection. In addition, DNA nanostructures used for sensing purposes have to withstand the different solution conditions while being intact during detection of biomarkers in vivo [67]. Specifically, these structures are prone to degradation by nucleases in biological systems. Recent chemical strategies that provide a solution to this problem include the use of a phosphorothioate backbone [68], locked nucleic acids (LNA) [69], L-DNA [70], 5'/3' modifications including hexaethylene glycol (HEG), hexane diol (C6) and $5^{\prime}$-phosphate (P) [71], and other xenonucleic acids [72]. Previous research has shown DNA nanostructures to be stable in cell lysates [73] and the integrity of these structures in tissue culture environment has been analyzed [74]. It would be useful to analyze the stability of these structures in a variety of environments (e.g., different cell types, normal versus tumorous tissues) so that the biosensors can be tailored for optimal responses. Future work on DNAbased chemical and biological sensors will especially aid in the characterization and development of these structures for in situ sensing. With recent developments in DNA-PAINT (a variation of point accumulation for imaging in nanoscale topography) $[75,76]$, it is possible to create DNA nanostructures that can signal the presence of specific biomarkers in vivo. Other recent developments in this front include DNA nanothermometers based on DNA clamp architectures that are useful for temperatures in the range of $30^{\circ} \mathrm{C}$ to $85^{\circ} \mathrm{C}$ [77]. In addition, DNA origami structures have been combined with solid-state nanopores for detection of $\lambda$-DNA molecules [78], opening up a new route to single molecule detection of biomolecules.

Self-assembly techniques, especially DNA origami, have made the construction of nanoscale objects easier. In addition, the cost involved in the preparation of DNA nanostructures has reduced in recent times with synthetic oligonucleotides being able to be simply ordered from a company.
Recent research has shown that the cost of synthetic DNA can be reduced further to as low as $\$ 0.001$ per base pair [79]. Moreover, custom-tailored DNA scaffolds now allow the construction of DNA origami structures of different sizes and are not limited to the frequently used M13 single strand [80]. With the aid of suitable purification methods [81-85], these nanostructures can be prepared in pure forms that provide enhanced sensitivity. DNA, being a biomolecule, also provides an advantage of being biocompatible [86] and can be useful for biosensing in combination with biomimetic approaches. Thus, designed DNA architectures provide a route to the creation of highly sensitive biosensors, with minimal cost and high assembly efficiency, with a selection of output strategies for varying purposes.

\section{Conflicts of Interest}

The author declares that there are no conflicts of interest regarding the publication of this paper.

\section{References}

[1] R. T. Ahuja and D. Kumar, "Recent progress in the development of nano-structured conducting polymers/nanocomposites for sensor applications," Sens. Actuators B, vol. 136, pp. 275-286, 2009.

[2] J. Lei and H. Ju, "Signal amplification using functional nanomaterials for biosensing," Chem. Soc. Rev, vol. 41, pp. 2122-2134, 2012.

[3] Y. Zhang, Y. Guo, Y. Xianyu, W. Chen, Y. Zhao, and X. Jiang, "Nanomaterials for ultrasensitive protein detection," $A d v$. Mater, vol. 25, pp. 3802-3819, 2013.

[4] W. Wang, T. Lin, S. Zhang, T. Bai, Y. Mi, and B. Wei, "Selfassembly of fully addressable DNA nanostructures from double crossover tiles," Nucleic Acids Research, vol. 44, no. 16, pp. 79897996, 2016.

[5] J. Zheng, J. J. Birktoft, Y. Chen et al., "From molecular to macroscopic via the rational design of a self-assembled 3D DNA crystal," Nature, vol. 461, pp. 74-77, 2009.

[6] D. Bhatia, S. Arumugam, M. Nasilowski et al., "Quantum dotloaded monofunctionalized DNA icosahedra for single-particle tracking of endocytic pathways," Nat. Nanotech, vol. 11, pp. 11121119, 2016.

[7] F. Zhang, S. Jiang, S. Wu et al., "Complex wireframe DNA origami nanostructures with multi-arm junction vertices," Nat. Nanotech, vol. 10, pp. 779-784, 2015.

[8] A. Czogalla, H. G. Franquelim, and P. Schwille, "DNA Nanostructures on Membranes as Tools for Synthetic Biology," Biophysical Journal, vol. 110, pp. 1698-1707, 2016.

[9] R. Chhabra, J. Sharma, Y. Liu, S. Rinker, and H. Yan, "DNA selfassembly for nanomedicine," Adv. Drug Deliv. Rev, vol. 62, pp. 617-625, 2010.

[10] C. M. Niemeyer, "Nanoparticles, proteins, and nucleic acids: biotechnology meets materials science," Angewandte Chemie International Edition, vol. 40, no. 22, pp. 4128-4158, 2001.

[11] J. Chao, D. Zhu, Y. Zhang, L. Wang, and C. Fan, "DNA nanotechnology-enabled biosensors," Biosens Bioelectron, vol. 76, pp. 68-79, 2016. 
[12] H. M. Meng, H. Liu, H. Kuai, R. Peng, L. Moa, and X. B. Zhang, "Aptamer-integrated DNA nanostructures for biosensing, bioimaging and cancer therapy," Chem. Soc. Rev, vol. 45, no. 9, pp. 2583-2602, 2016.

[13] A. R. Chandrasekaran, H. Wady, and H. K. K. Subramanian, "Nucleic acid nanostructures for chemical and biological sensing," Small, vol. 12, pp. 2689-2700, 2016.

[14] H. Pei, X. Zuo, D. Pan, J. Shi, Q. Huang, and C. Fan, "Scaffolded biosensors with designed DNA nanostructures," NPG Asia Materials, vol. 5, p. e51, 2013.

[15] J. Bath and A. J. Turberfield, "DNA nanomachines," Nat. Nanotech, vol. 2, pp. 275-284, 2007.

[16] F. C. Simmel and W. U. Dittmer, "DNA nanodevices," Small, vol. 1, pp. 284-299, 2005.

[17] A. Idili, A. Vallee-Belisle, and F. Ricci, "Programmable pHtriggered DNA nanoswitches," J. Am. Chem. Soc, vol. 136, pp. 5836-5839, 2014.

[18] S. Modi, M. G. Swetha, D. Goswami, G. D. Gupta, S. Mayor, and Y. Krishnan, "A DNA nanomachine that maps spatial and temporal pH changes inside living cells," Nature Nanotech, vol. 4, pp. 325-330, 2009.

[19] S. Chakraborty, S. Sharma, P. K. Maiti, and Y. Krishnan, “The poly dA helix: a new structural motif for high performance DNA-based molecular switches," Nucl. Acids Res, vol. 37, pp. 2810-2817, 2009.

[20] S. Burge, G. N. Parkinson, P. Hazel, A. K. Todd, and S. Neidle, "Quadruplex DNA: sequence, topology and structure," Nucl. Acids Res, vol. 34, pp. 5402-5415, 2006.

[21] S. Song, L. Wang, J. Li, C. Fan, and J. Zhao, "Aptamer-based biosensors," Trends Anal. Chem, vol. 27, pp. 108-117, 2008.

[22] S. Tombelli, M. Minunni, and M. Mascini, "Analytical applications of aptamers," Biosens. Bioelectron, vol. 20, pp. 2424-2434, 2005.

[23] B. Yurke, A. J. Turberfield, A. P. Mills Jr, F. C. Simmel, and J. L. Neumann, "A DNA-fuelled molecular machine made of DNA," Nature, vol. 406, pp. 605-608, 2000.

[24] S. Ranallo, M. Rossetti, K. W. Plaxco, A. Vallée-Bélisle, and F. Ricci, "A modular, DNA-based beacon for single-step fluorescence detection of antibodies and other proteins," Angew. Chem. Int, vol. 54, pp. 13214-13218, 2015.

[25] J. Liu and Y. Lu, "A colorimetric lead biosensor using DNAzyme-directed assembly of gold nanoparticles," J. Am. Chem. Soc, vol. 125, pp. 6642-6643, 2003.

[26] H. Pei, N. Lu, Y. Wen et al., "A DNA nanostructurebased biomolecular probe carrier platform for electrochemical biosensing," Adv Mater, vol. 22, pp. 4754-4758, 2010.

[27] A. R. Chandrasekaran, J. Zavala, and K. Halvorsen, "Programmable DNA Nanoswitches for Detection of Nucleic Acid Sequences," ACS Sensors, vol. 1, no. 2, pp. 120-123, 2015.

[28] A. Kuzuya, R. Watanabe, Y. Yamanaka, T. Tamaki, M. Kaino, and Y. Ohya, "Nanomechanical DNA origami pH sensors," Sensors, vol. 14, pp. 19329-19335, 2014.

[29] M. Li, J. Zhang, S. Suri, L. J. Sooter, D. Ma, and N. Wu, "Detection of adenosine triphosphate with an aptamer biosensor based on surface-enhanced Raman scattering," Anal Chem, vol. 84, pp. 2837-2842, 2012.

[30] D. Liu, A. Bruckbauer, C. Abell et al., "A reversible pH-driven DNA nanoswitch array," J. Am. Chem. Soc, vol. 128, pp. 20672071, 2006.

[31] X. M. Li, J. Song, T. Cheng, and P. Y. Fu, "A duplex-triplex nucleic acid nanomachine that probes $\mathrm{pH}$ changes inside living cells during apoptosis," Anal. Bioanal. Chem, vol. 405, pp. 59935999, 2013.

[32] X. Y. Li, J. Huang, H. X. Jiang, Y. C. Du, G. M. Hana, and D. M. Kong, "Molecular logic gates based on DNA tweezers responsive to multiplex restriction endonucleases," RSC Advances, vol. 6, no. 44, pp. 38315-38320, 2016.

[33] E. A. Jares-Erijman and T. M. Jovin, "FRET imaging," Nat. Biotech, vol. 21, pp. 1387-1395, 2003.

[34] S. Surana, J. M. Bhat, S. P. Koushika, and Y. Krishnan, "An autonomous DNA nanomachine maps spatiotemporal $\mathrm{pH}$ changes in a multicellular living organism," Nat. Commun, vol. 2, p. 340, 2011.

[35] S. Modi, C. Nizak, S. Surana, S. Halder, and Y. Krishnan, "Two DNA nanomachines map $\mathrm{pH}$ changes along intersecting endocytic pathways inside the same cell," Nat. Nanotech, vol. 8, pp. 459-467, 2013.

[36] H. Pei, L. Liang, G. Yao, J. Li, Q. Huang, and C. Fan, "Reconfigurable three-dimensional DNA nanostructures for the construction of intracellular logic sensors," Angew. Chem. Int, vol. 51, pp. 9020-9024, 2012.

[37] J. Y. Kim and J. S. Lee, "Synthesis and thermally reversible assembly of DNAgold nanoparticle cluster conjugates," Nano Lett, vol. 9, pp. 4564-4569, 2009.

[38] R. Elghanian, J. J. Storhoff, R. C. Mucic, R. L. Letsinger, and C. A. Mirkin, "Selective colorimetric detection of polynucleotides based on the distance-dependent optical properties of gold nanoparticles," Science, vol. 277, pp. 1078-1081, 1997.

[39] P. Lie, J. Liu, Z. Fang, B. Dun, and L. Zeng, "A lateral flow biosensor for detection of nucleic acids with high sensitivity and selectivity," Chemical Communications, vol. 48, no. 2, pp. 236238, 2012.

[40] G. Zhou, M. Lin, P. Song, X. Chen et al., "Multivalent capture and detection of cancer cells with DNA nanostructured biosensors and multibranched hybridization chain reaction amplification," Anal. Chem, vol. 86, pp. 7843-7848, 2014.

[41] H. Pei, Y. Wan, J. Li et al., "Regenerable electrochemical immunological sensing at DNA nanostructure-decorated gold surfaces," Chem. Commun, vol. 47, pp. 6254-6256, 2011.

[42] X. Chen, C. Y. Hong, Y. H. Lin, J. H. Chen, G. N. Chen, and H. H. Yang, "Enzyme-free and label-free ultrasensitive electrochemical detection of human immunodeficiency virus DNA in biological samples based on long-range self-assembled DNA nanostructures," Anal. Chem, vol. 84, pp. 8277-8283, 2012.

[43] A. A. Lubin and K. W. Plaxco, "Folding-based electrochemical biosensors: the case for responsive nucleic acid architectures," Acc. Chem. Res, vol. 43, pp. 496-505, 2010.

[44] H. Li, N. Arroyo-Currás, D. Kang, F. Ricci, and K. W. Plaxco, "Dual-Reporter drift correction to enhance the performance of electrochemical aptamer-based sensors in whole blood," Journal of the American Chemical Society, vol. 138, no. 49, pp. 1580915812, 2016.

[45] B. S. Ferguson, D. A. Hoggarth, D. Maliniak et al., "Real-time, aptamer-based tracking of circulating therapeutic agents in living animals," Science Translational Medicine, vol. 5, Article ID 213ral65, 2013.

[46] A. J. Bonham, N. G. Paden, F. Ricci, and K. W. Plaxco, "Detection of IP-10 protein marker in undiluted blood serum via an electrochemical E-DNA scaffold sensor," Analyst, vol.138, pp. 5580-5583, 2013.

[47] A. Vallée-Bélisle, F. Ricci, T. Uzawa, F. Xia, and K. W. Plaxco, "Bioelectrochemical switches for the quantitative detection of 
antibodies directly in whole blood," J. Am. Chem. Soc, vol. 134, pp. 15197-15200, 2012.

[48] M. A. Koussa, K. Halvorsen, A. Ward, and W. P. Wong, "DNA nanoswitches: a quantitative platform for gel-based biomolecular interaction analysis," Nat. Methods, vol. 12, pp. 123-126, 2015.

[49] P. W. K. Rothemund, "Folding DNA to create nanoscale shapes and patterns," Nature, vol. 440, pp. 297-302, 2006.

[50] Y. Ke, S. Lindsay, Y. Chang, Y. Liu, and H. Yan, "Self-assembled water-soluble nucleic acid probe tiles for label-free RNA hybridization assays," Science, vol. 319, pp. 180-183, 2008.

[51] H. K. K. Subramanian, B. Chakraborty, R. Sha, and N. C. Seeman, "The label-free unambiguous detection and symbolic display of single nucleotide polymorphisms on DNA origami," Nano Lett, vol. 11, pp. 910-913, 2011.

[52] K. Kneipp, Y. Wang, H. Kneipp et al., "Single molecule detection using surface-enhanced raman scattering (SERS)," Phys. Rev. Lett, vol. 78, pp. 1667-1670, 1997.

[53] K. L. Kelly, E. Coronado, L. L. Zhao, and G. C. Schatz, "The optical properties of metal nanoparticles: the influence of size, shape, and dielectric environment," J. Phys. Chem. B, vol. 107, no. particles, pp. 668-677, 2003.

[54] B. Küstner, M. Gellner, M. Schütz et al., "SERS labels for red laser excitation: silica-encapsulated SAMs on tunable gold/silver nanoshells," Angew. Chem. Int, vol. 48, pp. 1950-1953, 2009.

[55] W. E. Doering and S. Nie, "Spectroscopic tags using dyeembedded nanoparticles and surface-enhanced raman scattering," Anal. Chem, vol. 75, pp. 6171-6176, 2003.

[56] Y. C. Cao, R. Jin, C. S. Thaxton, and C. A. Mirkin, "A twocolor-change, nanoparticle-based method for DNA detection," Talanta, vol. 67, pp. 449-455, 2005.

[57] J. W. Keum, M. Kim, J. M. Park, C. Yoo, N. Huh, and S. C. Park, "DNA-directed self-assembly of three-dimensional plasmonic nanostructures for detection by surface-enhanced Raman scattering (SERS)," Sensing and Bio-Sensing Research, vol. 1, pp. 21-25, 2014.

[58] V. V. Thacker, L. O. Herrmann, D. O. Sigle et al., "DNA origami based assembly of gold nanoparticle dimers for surfaceenhanced Raman scattering," Nat. Commun, vol. 5, article 3448, 2014.

[59] O. I. Wilner and I. Willner, "Functionalized DNA nanostructures," Chem. Rev, vol. 112, pp. 2528-2556, 2012.

[60] A. R. Chandrasekaran, "Programmable DNA scaffolds for spatially-ordered protein assembly," Nanoscale, vol. 8, pp. 44364446, 2016.

[61] D. A. Rusling, A. R. Chandrasekaran, Y. P. Ohayon et al., "Functionalizing designer DNA crystals with a triple-helical veneer," Angew. Chem. Int, vol. 53, pp. 3979-3982, 2014.

[62] V. Valsangkar, A. R. Chandrasekaran, R. Wang et al., "Clickbased functionalization of a 2 ' -O-propargyl-modified branched DNA nanostructure," J. Mater. Chem. B, vol. 5, no. 11, pp. 20742077, 2017.

[63] C. H. Lu and I. Willner, "Stimuli-responsive DNA-functionalized nano-/microcontainers for switchable and controlled release," Angew. Chem. Int, vol. 54, pp. 12212-12235, 2015.

[64] X. Yang, Y. Tang, S. D. Mason, J. Chen, and F. Li, "Enzymepowered three-dimensional DNA nanomachine for DNA walking, payload release, and biosensing," ACS Nano, vol. 10, pp. 2324-2330, 2016.

[65] N. Chen, S. Qin, X. Yang, Q. Wang, J. Huang, and K. Wang, "“Sense-and-Treat" DNA nanodevice for synergetic destruction of circulating tumor cells," ACS Applied Materials \& Interfaces, vol. 8, no. 40, pp. 26552-26558, 2016.

[66] D. Li, W. Cheng, Y. Li et al., "Catalytic hairpin assembly actuated DNA nanotweezer for logic gate building and sensitive enzymefree biosensing of microRNAs," Analytical Chemistry, vol. 88, no. 15, pp. 7500-7506, 2016.

[67] D. S. Lee, H. Qian, C. Y. Tay, and D. T. Leong, "Cellular processing and destinies of artificial DNA nanostructures," Chemical Society Reviews, vol. 45, no. 15, pp. 4199-4225, 2016.

[68] J. J. Fakhoury, C. K. McLaughlin, T. W. Edwardson, J. W. Conway, and H. F. Sleiman, "Development and characterization of gene silencing DNA cages," Biomacromolecules, vol. 15, pp. 276-282, 2014.

[69] T. Shimo, K. Tachibana, K. Saito et al., "Design and evaluation of locked nucleic acid-based splice-switching oligonucleotides in vitro," Nucl. Acids Res, pp. 8174-8187, 2014.

[70] C. Lin, Y. Ke, Z. Li, J. H. Wang, Y. Liu, and H. Yan, "Mirror image DNA nanostructures for chiral supramolecular assemblies," Nano Lett, vol. 9, pp. 433-436, 2009.

[71] J. W. Conway, C. K. McLaughlin, K. J. Castor, and H. Sleiman, "DNA nanostructure serum stability: greater than the sum of its parts," Chem. Commun, vol. 49, pp. 1172-1174, 2013.

[72] V. B. Pinheiro and P. Holliger, "Towards XNA nanotechnology: new materials from synthetic genetic polymers," Trends Biotechnol, vol. 32, pp. 321-328, 2014.

[73] Q. Mei, X. Wei, F. Su et al., "Stability of DNA origami nanoarrays in cell lysate," Nano Lett, vol. 11, pp. 1477-1482, 2011.

[74] J. Hahn, S. F. J. Wickham, W. M. Shih, and S. D. Perrault, "Addressing the instability of DNA nanostructures in tissue culture," ACS Nano, vol. 8, pp. 8765-8775, 2014.

[75] C. Lin, R. Jungmann, A. M. Leifer et al., "Sub-micrometer geometrically encoded fluorescent barcodes self-assembled from DNA," Nat. Chem, vol. 4, pp. 832-839, 2012.

[76] B. J. Beliveau, A. N. Boettiger, M. S. Avendaño et al., "Singlemolecule super-resolution imaging of chromosomes and in situ haplotype visualization using Oligopaint FISH probes," Nat. Commun, vol. 6, article 7147, 2015.

[77] D. Gareau, A. Desrosiers, and A. Vallée-Bélisle, "Programmable quantitative DNA nanothermometers," Nano Lett, vol. 16, pp. 3976-3981, 2016.

[78] N. A. W. Bell, C. R. Engst, M. Ablay et al., DNA Origami Nanopores. Nano Lett, vol. 12, pp. 512-517, 2012.

[79] A. N. Marchi, I. Saaem, B. N. Vogen, S. Brown, and T. H. LaBean, “Toward larger DNA origami," Nano Lett, vol. 14, pp. 5740-5747, 2014.

[80] A. R. Chandrasekaran, M. Pushpanathan, and K. Halvorsen, Evolution of DNA origami scaffolds. Mat. Lett, vol. 170, pp. 221224, 2016.

[81] C. Lin, S. D. Perrault, M. Kwak, F. Graf, and W. M. Shih, "Purification of DNA-origami nanostructures by rate-zonal centrifugation," Nucleic Acids Research, vol. 41, no. 2, p. e40, 2013.

[82] A. Shaw, E. Benson, and B. Högberg, "Purification of functionalized DNA origami nanostructures," ACS Nano, vol. 9, pp. 4968-4975, 2015.

[83] E. Stahl, T. G. Martin, F. Praetorius, and H. Dietz, "Facile and scalable preparation of pure and dense DNA origami solutions," Angew. Chem. Int, vol. 53, pp. 12735-12740, 2014.

[84] G. Bellot, M. A. McClintock, C. Lin, and W. M. Shih, "Recovery of intact DNA nanostructures after agarose gel-based separation," Nat. Methods, vol. 8, pp. 192-194, 2011. 
[85] K. Halvorsen, M. Kizer, X. Wang, A. R. Chandrasekaran, and M. Basanta Sanchez, "Shear dependent LC purification of an engineered DNA nanoswitch and implications for DNA origami," Anal. Chem, 2017.

[86] A. R. Chandrasekaran, N. Anderson, M. Kizer, K. Halvorsen, and X. Wang, "Beyond the fold: Emerging biological applications of DNA origami," ChemBioChem, vol. 17, pp. 1081-1089, 2016. 

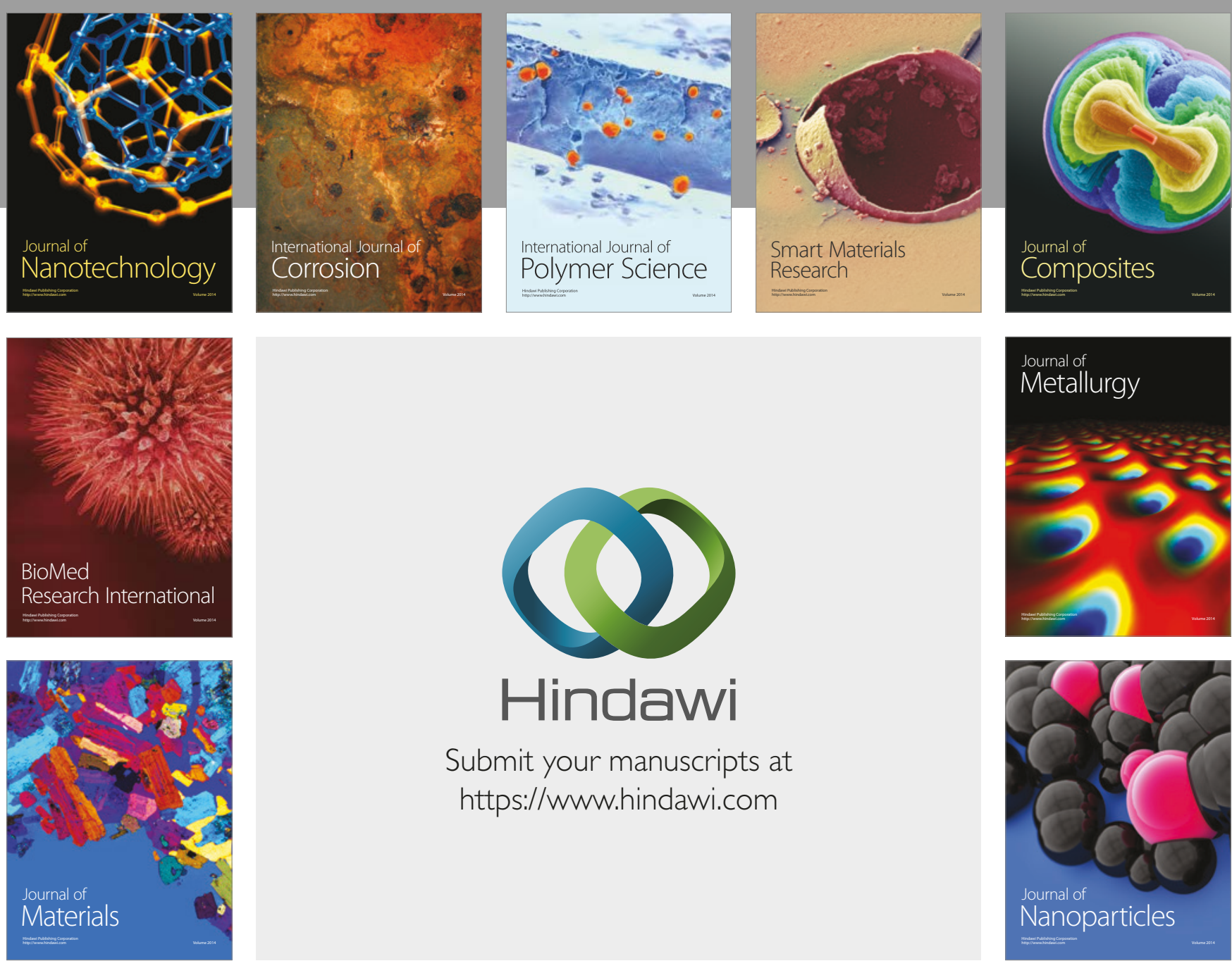

\section{Hindawi}

Submit your manuscripts at

https://www.hindawi.com
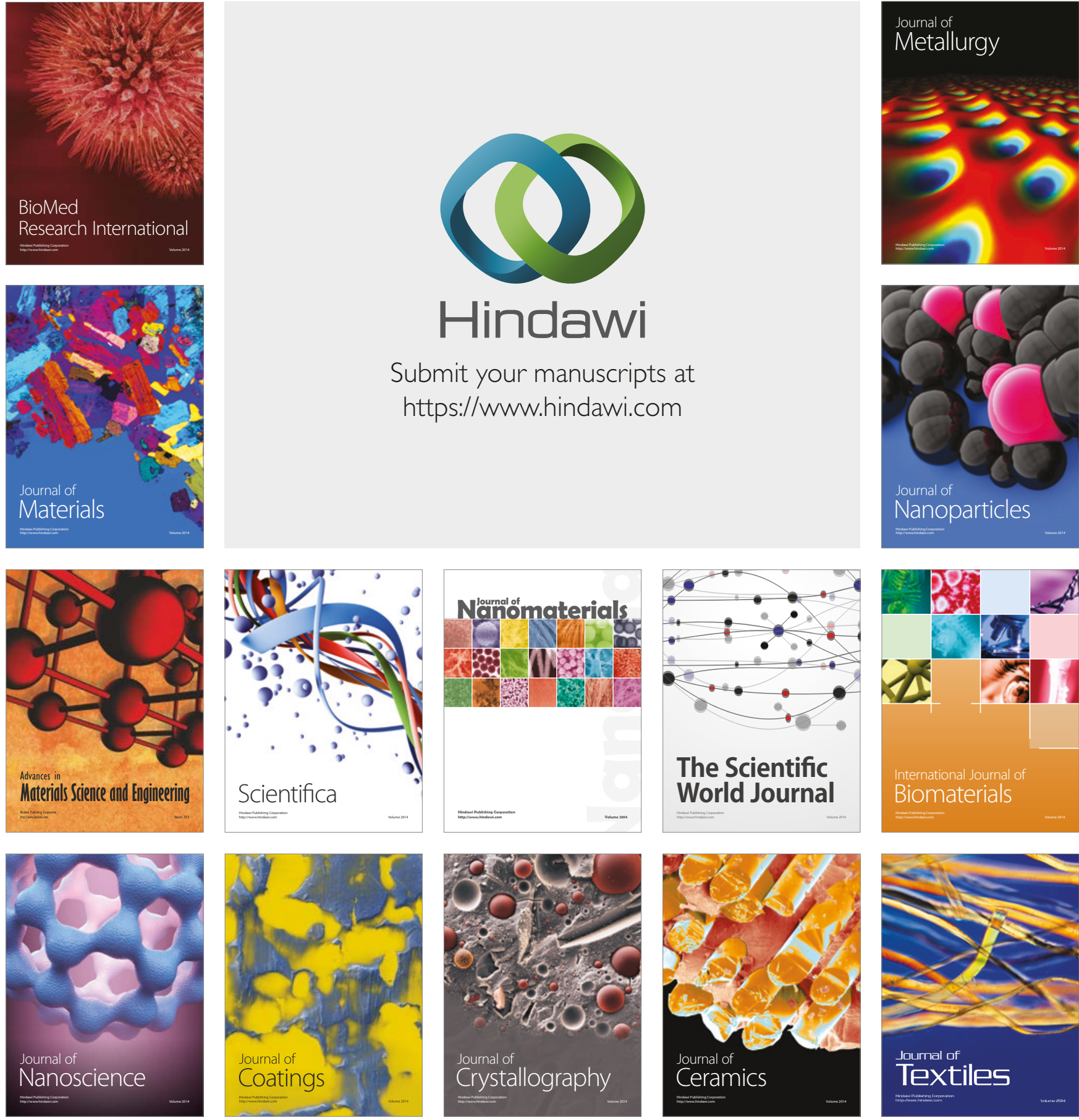

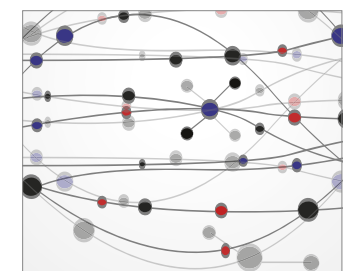

The Scientific World Journal
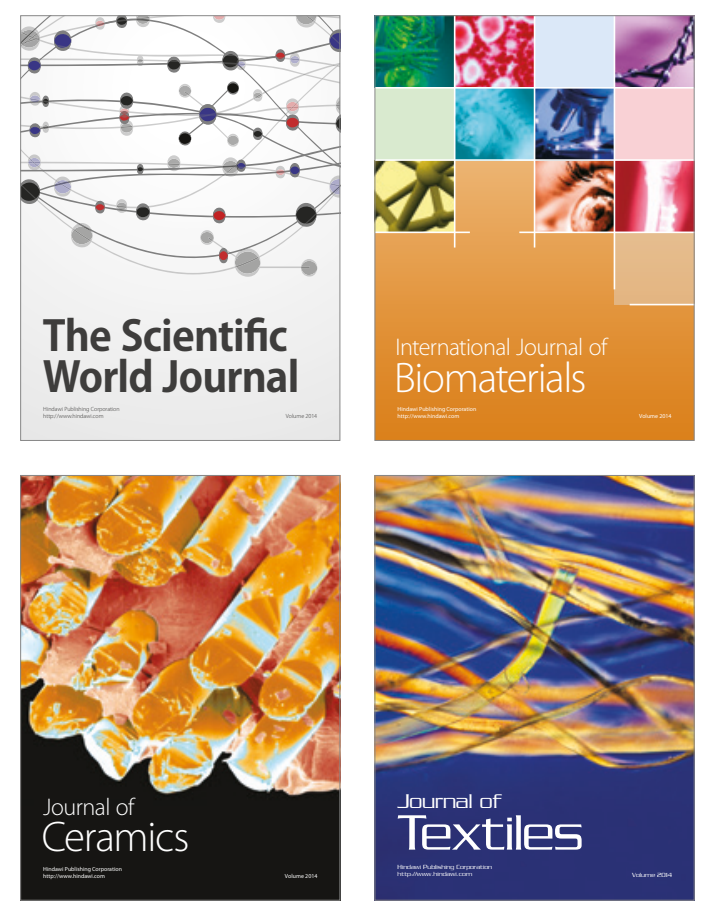